\title{
Primary measurement of total ultrasonic power with improved accuracy in rf voltage measurement
}

\author{
P. K. Dubey, ${ }^{\text {a) }}$ Ashok Kumar, Yudhisther Kumar, Reeta Gupta, and Deepa Joshi \\ Acoustics and Ultrasonic Standards, National Physical Laboratory, New Delhi 110012, India
}

(Received 18 March 2010; accepted 2 August 2010; published online 20 October 2010)

\begin{abstract}
Out of the various existing ultrasonic power measurement techniques, the radiation force balance method using microbalance is most widely used in low power (below $1 \mathrm{~W}$ ) regime. The major source of uncertainty associated with this technique is the error in ac voltage measurement applied to the transducer for the generation of ultrasonic waves. The sources that deteriorate the ac voltage measurement accuracy include cable length and impedance mismatch. We introduce a new differential peak to peak measurement approach to reduce the ac voltage measurement error. The method holds the average peak amplitude of each polarity. Ultralow offset difference amplifier is used to measure peak to peak voltage. The method is insensitive to the variations in the dc offset of the source. The functionality of this method has been tested and compared with the conventional rf voltage measurement method. The output of this proposed technique is dc, which can be measured with an error of less than 0.1\%. (C) 2010 American Institute of Physics. [doi:10.1063/1.3482157]
\end{abstract}

\section{INTRODUCTION}

The use of ultrasound in medicine has increased dramatically in the recent years. It is however generally restricted to the frequency range of $0.5-15 \mathrm{MHz}$. The lower limit is dictated by the velocity of ultrasound in tissue (approximately $1500 \mathrm{~m} / \mathrm{s}$ ), which makes the wavelength, and hence the resolution or localization at lower frequencies unacceptable. The upper limit is determined by the attenuation of ultrasound that increases with an increase in frequency. ${ }^{1-5}$ Measurement of ultrasonic power from a medical ultrasonic equipment is essential for the assessment of the performance or safety of that equipment. ${ }^{5,6}$ The power generated from medical transducer ranges from a few milliwatts for diagnostic equipment to several watts for physiotherapy applications. ${ }^{7-10}$

In the design and optimization of new ultrasonic equipment, it is often essential to determine acoustic output, as it plays an important role in the ultimate performance level of any particular system. Absolute measurement techniques provide accurate reference information and the results of measurements are capable of being monitored over both short and long periods, forming the basis of objective in national and international comparison and assessment. ${ }^{3}$ The time average ultrasonic power emitted by the source transducer is one of the key values taken into account. ${ }^{11}$ According to both fundamental International Electrotechnical Commission (IEC) standards dealing with acoustic output of medical ultrasonic devices, that of diagnostic devices (IEC 61157) (Ref. 12) and that of therapeutic devices (IEC 61689), ${ }^{13}$ the ultrasonic power is to be measured and declared.

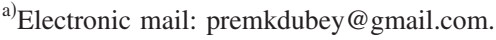

The technique recommended in IEC 61161 uses a radiation force balance. ${ }^{14}$ An appropriately constructed target, properly aligned in a steady state under ultrasound field, is subjected to a radiation force given by ${ }^{15-17}$

$$
F=W / c(T),
$$

where $W$ is the time averaged spatially integrated power intercepted by the target and $c$ is the propagation velocity of ultrasound in the medium used as a function of temperature. ${ }^{18-20}$ Time averaging of ultrasound occurs because under practical circumstances, the inertia of the target causes it to effectively integrate pulses into a corresponding steady state force. Spatial integration is exploited by using targets larger in cross section than the incident beam, ${ }^{16}$

$$
W=\Delta m g c(T),
$$

where $\Delta m$ is the measured weight change caused by the radiation force and $g$ is the value of gravity at the experimental site. The radiation conductance of the ultrasonic transducer is given by ${ }^{7}$

$$
\begin{aligned}
& G=\frac{W}{V_{\mathrm{rms}}^{2},} \\
& G=\frac{\Delta m g c(T)}{V_{\mathrm{rms}}^{2}},
\end{aligned}
$$

where $V_{\mathrm{rms}}$ is the root mean square voltage applied to the transducer. Hence more accurate measurement of $G$ requires more accurate measurement of all four parameters of Eq. (4). At the National Physical Laboratory, India (NPLI), the measurement of change in weight is measured by a highly sensitive microbalance [Sartorius (Goettingen, Germany), model: $21 \mathrm{~S}]$, having a measurement resolution of $1 \mu \mathrm{g} .{ }^{21}$ The value of $g$ has been actually measured (accurate up to sixth decimal place) by the survey of India at station 9 (the experimental site) of the main building of NPL, where the 
actual power measurements are carried out. Ultrasonic propagation velocity at the measured temperature is taken from the standard literature. ${ }^{18,19}$

Accurate measurement of rf voltage applied across the transducer is one of the critical problems associated with this system. Generally, the ac voltage applied to the transducer is directly measured with the help of an ac voltmeter or a digital oscilloscope. ${ }^{8,22}$ It is suggested in Ref. 22 that input impedance of the measuring device should be at least 100 times larger than the electrical impedance of the transducer and should be measured with an accuracy better than $\pm 1 \%$. High input impedance is particularly required to reduce the error in measurement caused by the series inductive effect in the connecting cable at high frequencies. Another approach and perhaps the most accurate method to measure the rf voltage is to use the thermal converters. ${ }^{23-25}$ The basic principle of thermal converter is the use of a nonreactive high frequency resistor to convert the electrical energy into thermal energy. The thermal energy is sensed by a temperature sensor directly attached to the resistor (heater). ${ }^{26-28}$ Although thermal converters are appropriate to measure rf voltage more accurately, still, they are particularly developed to measure lower voltages (less than $5 \mathrm{~V}$ ) and offer low input impedance. ${ }^{24,25}$ The range of measurement is increased by using a series resistance of suitable value and appropriate tolerance. However, the tolerance of this resistor deteriorates the measurement accuracy. In addition to this, the thermal converters have poor dynamic response. ${ }^{23,24}$ This makes it inconvenient for an automatic system, where quick ultrasonic response of the transducer is to be measured at higher voltages.

In this article the error caused in the ac voltage measurement due to voltage drop across the series inductance and shunt capacitance offered by the coaxial cable ${ }^{29}$ is discussed. The measurement accuracy is the function of frequency and deteriorates at higher frequency. We introduce a differential peak to peak measurement circuit that is directly applied at the transducer without coaxial cable. The output of this module is pure dc which is connected to a digital storage oscilloscope [Tektronix (Beaverton, USA) Digital Storage Oscilloscope (DSO), model: TDS210] for measurement. As the module provides dc the voltage drop in the coaxial cable is negligible and hence improves the uncertainty associated with the rf voltage measurement. The circuit described here has a fast dynamic response, and hence it is suitable for an automated ultrasonic power measurement setup.

\section{RADIATION FORCE BALANCE SETUP AT NPLI}

Figure 1 shows the block diagram of the radiation force balance (RFB) system. The water bath is made up of Perspex with its inner length, breadth, and height as 300, 300, and $170 \mathrm{~mm}$, respectively. The water bath has a removable lid by which the bath is covered after fixing the transducer and hanging the absorbing target. The specially designed polyurethane polymer is used as an absorbing target. ${ }^{6}$ The target dimensions are $50 \mathrm{~mm} \times 60 \mathrm{~mm} \times 15 \mathrm{~mm}$. An LM 35 sensor $^{30}$ based digital temperature monitor designed in the laboratory is used for the measurement of water bath temperature with $\pm 0.2{ }^{\circ} \mathrm{C}$ accuracy. The propagation velocity of

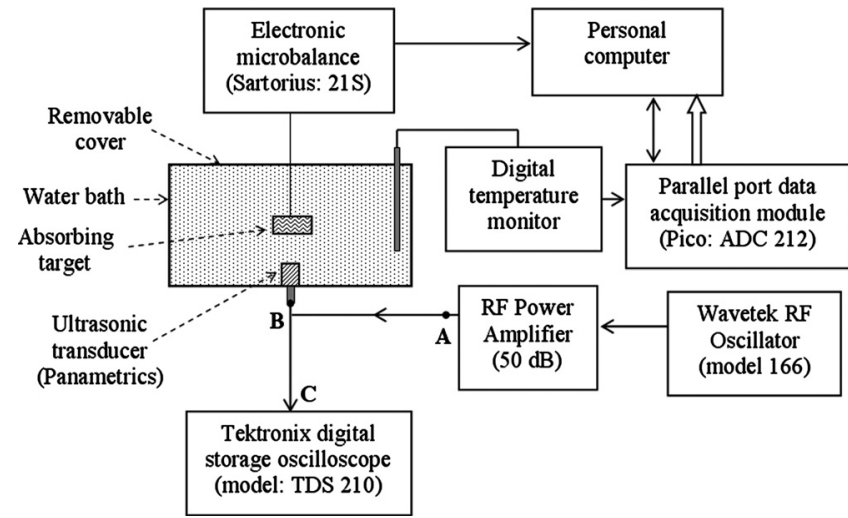

FIG. 1. Block diagram showing the parts of radiation force balance setup at NPL, India, used to measure total ultrasonic power of a transducer.

ultrasound is taken at the measured temperature using standard literature. ${ }^{18,19}$ Highly sensitive microbalance is used to measure the change in the weight of the absorber. The microbalance is interfaced to the personal computer via RS 232 port for its data acquisition. The transducer is excited at the desired voltage and frequency using Wavetek function generator (model: 166) and a fixed $50 \mathrm{~dB}$ power amplifier. A coaxial cable of about $1 \mathrm{~m}$ is used at the output of amplifier to connect the transducer. The input voltage to the transducer is measured with the help of a digital storage oscilloscope.

\section{EFFECT OF COAXIAL CABLE ON VOLTAGE MEASUREMENT ACCURACY}

The highly sensitive RFB setup used in ultrasound power measurement is not portable. So, cables $\mathrm{AB}$ and $\mathrm{BC}$ shown in Fig. 1 are unavoidable. Figure 2 shows the radio frequency equivalent circuit of the voltage measurement system used in RFB. Here in this figure, series inductance and shunt capacitance of cable are only shown as they have major roles at radio frequencies.

Rs is the output impedance of signal sources L1, C1, and $\mathrm{L} 2$, and $\mathrm{C} 2$ are the series inductance and parallel capacitances offered by the coaxial cables used in the measurement. Cc is the capacitance offered by T type BNC (Bayonet Neill-Concelman) connector used at the transducer. $\mathrm{Zt}$ is the impedance offered by the piezoelectric transducer under test.

It is common to measure the voltage applied to the transducer at point A (see Fig. 1). ${ }^{7,16,31}$ This is performed by using a splitting $\mathrm{T}$ connector at the output of the source and measured with the help of coaxial cable. This will have better accuracy at the lower range of frequencies (typically

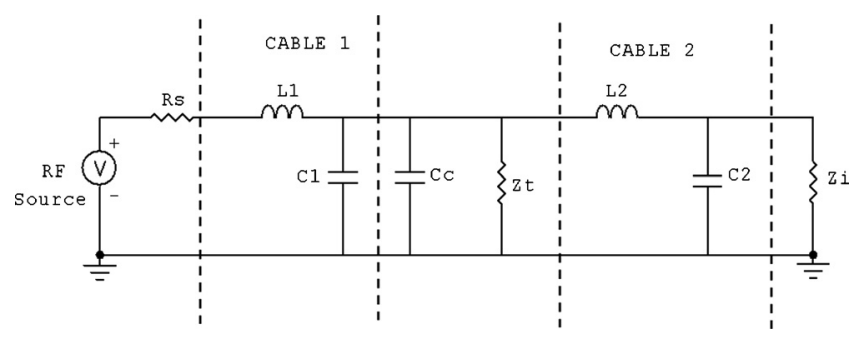

FIG. 2. Equivalent circuit showing different components that affect the voltage measurement accuracy. 


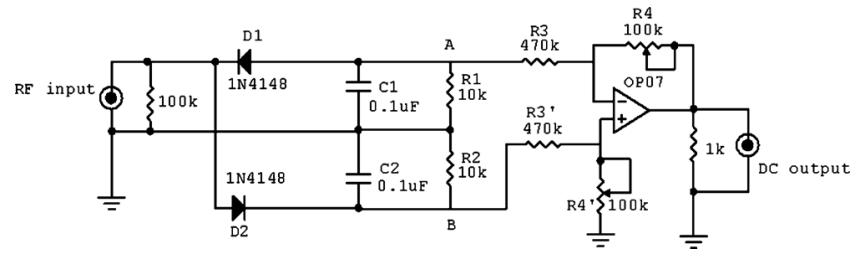

FIG. 3. Circuit diagram of the developed differential peak to peak measurement module.

$0.5-5 \mathrm{MHz}$ ). If the excitation frequency is increased, the potential difference between points A and B (Fig. 1) increases, which results in the deterioration of the ac voltage measurement accuracy. Hence, it will be a wise decision to measure voltage directly at point B using cable 2 , as shown in Fig. 2. If the input impedance of the measuring instrument is low, the voltage drop across the series inductive element increases, which results in an increase in error in the measurement.

\section{DIFFERENTIAL PEAK TO PEAK MEASUREMENT APPROACH}

The error in voltage measurement caused by coaxial cable can be minimized to a great extent using a rectifier module or a thermal converter that is directly connected to the transducer. The rectifier converts ac into dc and feeds to the dc voltage measuring instrument.

Keeping the above critical problem in view, a differential peak to peak measurement (DPPM) module has been developed to minimize the rf voltage measurement error. Figure 3 shows the circuit diagram of this module, which is directly attached at the transducer node (point B in Fig. 1) without using a cable.

The fast switching diodes (1N 4148) D1 and D2 conduct for the negative and positive half cycle of the input signal, respectively. The RC time constant of $1 \mathrm{~ms}$ is selected, which is at least a thousand times more than the time period of the input $\mathrm{rf}$ voltage. As the rf voltage applied to the transducer is a continuous sinusoidal signal, points A and B hold the peak negative and positive values, respectively. Voltages at points $\mathrm{A}$ and $\mathrm{B}$ are given by the equations

$$
\begin{aligned}
& V_{A}=V_{\mathrm{np}}+V_{D}, \\
& V_{B}=V_{\mathrm{pp}}-V_{D},
\end{aligned}
$$

where $V_{\mathrm{np}}$ is the negative peak value of the input signal and taken as negative, $V_{\mathrm{pp}}$ is the positive peak value of the input signal and taken as positive, and $V_{D}$ is the voltage drop across the diode while conducting.

Ultralow offset, high input impedance operational amplifier (OP07) (Ref. 32) is used as a difference amplifier with an attenuation factor of 10 . The resistors $\mathrm{R} 4$ and $\mathrm{R} 4{ }^{\prime}$ are trimmed to achieve the desired attenuation factor. The amplifier amplitude is taken in the span of $0-10 \mathrm{~V}$ and hence covering the ac input measurement range of $0-100 \mathrm{~V}$ peak to peak.

The output voltage of the Operational Amplifier (opamp) is given by

$$
V_{o}=\frac{R_{4}}{R_{3}}\left(V_{B}-V_{A}\right) .
$$

From Eqs. (5) and (6) and R4, R3 for the attenuation factor of 10 , we get

$$
V_{o}=\frac{\left(V_{\mathrm{pp}}-V_{\mathrm{np}}\right)-2 V_{D}}{10} .
$$

For silicon diode, $V_{D}=0.7 \mathrm{~V}$. Equation (7) shows that the dc output voltage is proportional to the peak to peak rf voltage. It is also clear that the output is independent of the dc offset present in the input voltage. For instance, $1 \mathrm{~V}$ positive dc offset in the rf input signal increases $V_{\mathrm{pp}}$ by $1 \mathrm{~V}$ and reduces $V_{\mathrm{np}}$ by the same amount $(1 \mathrm{~V})$. Thus, it is canceled out by the difference amplifier.

\section{A. Temperature effects on DPPM circuit}

The admissible power dissipation of $1 \mathrm{~N} 4148$ diode $^{33}$ depends on ambient temperature, and it is seen that it allows maximum dissipation $(500 \mathrm{~mW})$ until $30{ }^{\circ} \mathrm{C}$ and then gradually reduces (restricts) as the temperature is increased. It is also obvious from the circuit that the charging current through the diode will be initially higher during the first cycle while charging a fully discharged capacitor. It gradually reduces in the successive input cycles. This avoids the increase of junction temperature of the diode, which in turn minimizes the effect of temperature over it.

In case of an operational amplifier, the variation in temperature causes $\mathrm{drift}^{32}$ in the input offset voltage $\left(1.3 \mu \mathrm{V} /{ }^{\circ} \mathrm{C}\right)$ as well as in the input offset current $\left(35 \mathrm{pA} /{ }^{\circ} \mathrm{C}\right)$. However, this effect is negligible since the opamp used is at large signal levels (a few volts) and the gain is less than unity. Also to minimize the temperature effects on the ultrasonic power measurement setup, the laboratory room is air conditioned and the temperature is maintained at $23{ }^{\circ} \mathrm{C} \pm 1{ }^{\circ} \mathrm{C}$.

\section{RESULT AND DISCUSSION}

The variation of the input impedance of DSO (Tektronics: TDS 210) has been measured and compared with that of developed detector. The impedance has been calculated by connecting the source directly to channel A of DSO and a pure known resistance $(1 \mathrm{k} \Omega)$ in series with channel $\mathrm{B}$ of DSO. The impedance of channel B is calculated by knowing the drop across channel B. Similarly the input impedance of the DPPM module is calculated by connecting the module with channel B. At each frequency the parallel effect of module and DSO impedance was taken into account to calculate the DPPM module input impedance. Figure 4 shows the variation of DSO input impedance as a function of frequency. Initially, at $500 \mathrm{kHz}$ the input impedance $(\mathrm{Zi})$ observed is high; about $30 \mathrm{k} \Omega$. Above $20 \mathrm{MHz} \mathrm{Zi}$ decreases (100 times less) to about $300 \Omega$. Such a wide change in $\mathrm{Zi}$ causes variation at the input to DSO, consequently measuring the voltage that exactly does not appear across the transducer. As can be seen in Fig. 4, the input impedance offered by the developed module shows an almost flat response of the input impedance. It shows about $2.5 \mathrm{k} \Omega$ impedance from 


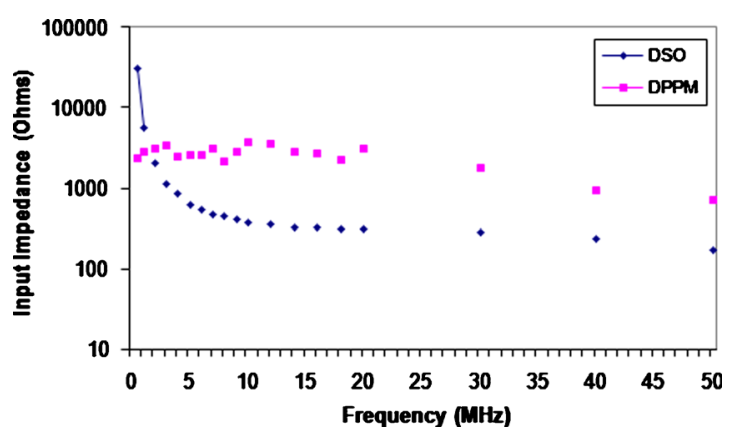

FIG. 4. (Color online) Variation of DSO and DPPM module impedance as a function of frequency.

$500 \mathrm{kHz}$ to $20 \mathrm{MHz}$ (covering entire range of medical transducers). As the module impedance is higher so, it hardly loads the source. Furthermore, there is no cable between the transducer and the module. The transducer voltage is directly fed as an input to the module.

In order to study the voltage linearity response of the developed module, the output has been recorded for each step of input voltage at $10 \mathrm{MHz}$. The rf voltage was applied and measured by DSO without cable (directly mounted on DSO input using BNC T connector). The typical linearity response along with the linear fit curve is shown in Fig. 5. The comparison with the linear fit equation $(y=m x+c)$ shows a slope of 0.1013 and is due to the attenuation factor of difference amplifier. The constant 0.1312 is due to the voltage drop of both the diodes.

The frequency response of the developed module has also been studied and is shown in Fig. 6. The output of the module was measured at 15 frequency points. Each time rf input was adjusted to $10 \mathrm{~V}_{\text {p.p. }} \pm 1 \%$ as it varies with change in frequency. The output voltage of the module was measured with a digital oscilloscope, which has measurement accuracy within $\pm 1 \%$. The figure clearly depicts that the variation in the measured voltage is within $\pm 1 \%$. Hence it appears that the scatter in the dc output voltage in Fig. 6 is due to the limitation of DSO.

The technique of DPPM has also been compared with the conventional direct $\mathrm{rf}$ measurement approach. Figure 7 shows the variation of total acoustic power versus frequency of a $10 \mathrm{MHz}$ immersion transducer (Panametrics). The voltage monitored in both cases was the same $\left(20 \mathrm{~V}_{\text {p.p. }}\right)$. From the figure it is clear that initially up to $8 \mathrm{MHz}$, the actual voltages (across transducer) are almost equal, but above

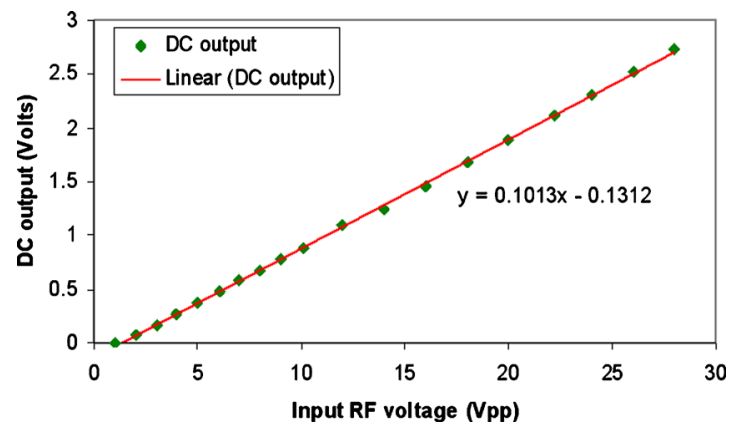

FIG. 5. (Color online) Linearity response of the DPPM circuit.

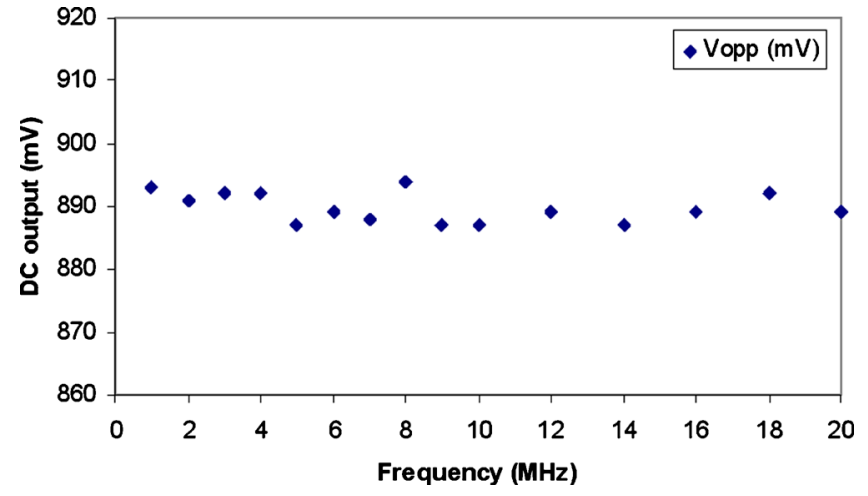

FIG. 6. (Color online) Variation of output de voltage of the DPPM circuit at various frequencies.

$9 \mathrm{MHz}$, the rf measurement method shows lower output power. This clearly demonstrates that even though the monitored voltage in both the cases were same $\left(20 \mathrm{~V}_{\text {p.p. }}\right)$, the voltage actually experienced by the transducer in $\mathrm{rf}$ approach was lower than in the developed dual peak method. This is particularly true above $8 \mathrm{MHz}$, where the reactance offered by the inductive component of cable increases, thereby reducing the voltage across transducer.

\section{CONCLUSION}

A peak to peak measurement module has been successfully designed and its functionality realized with a view to minimize the error in voltage measurement in the ultrasonic power evaluation. It has been found to be better and more accurate than measuring the rf voltage directly. As the circuit employs a very fast diode and peak detector, it has fast dynamic response. It holds the dc voltage within one complete input $\mathrm{rf}$ cycle. Such fast technique is necessarily needed while measuring ultrasonic power above few hundreds of milliwatts, where the temperature of the absorber may increase if the measurement takes longer time.

The dc output obtained from the developed module needs to be measured with higher accuracy. This ultimately increases the measurement accuracy of rf voltage. Available literature on voltage measurement ${ }^{34}$ reveals that dc voltage measurement is possible with accuracy better than $0.1 \%$. Furthermore, as the output of DPPM circuit is dc, a coaxial cable can be used to couple output to a high accuracy data

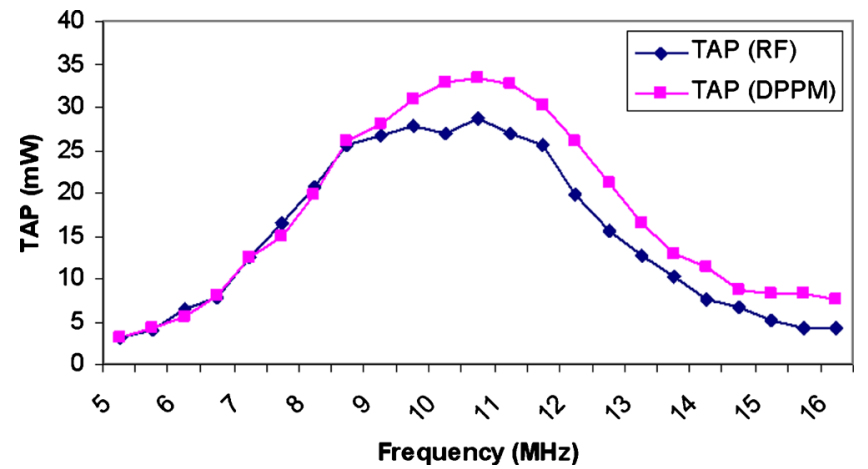

FIG. 7. (Color online) Variation of total acoustic power vs frequency of a $10 \mathrm{MHz}$ immersion transducer. 
acquisition system. ${ }^{35}$ It has also been shown that this technique is not affected by the variation in the dc offset present in the signal source.

${ }^{1}$ E. M. Schafer and P. A. Lewin, IEEE Trans. Ultrason. Ferroelectr. Freq. Control 35, 102 (1988).

${ }^{2}$ K. Martin, IEEE Trans. Ultrason. Ferroelectr. Freq. Control 35, 140 (1988).

${ }^{3}$ R. C. Preston and A. J. Livett, IEE Proc., Part A: Phys. Sci., Meas. Instrum., Manage. Educ. 131, 233 (1984).

${ }^{4}$ K. G. Lewis, Jr. and W. L. Olbricht, Rev. Sci. Instrum. 79, 114302 (2008).

${ }^{5}$ E. B. Miller and D. G. Eitzen, IEEE Trans. Sonics Ultrason. SU-26, 28 (1979).

${ }^{6}$ P. A. Lewin, N. Barrie-Smith, M. Ide, K. Hynynen, and M. Macdonald, J. Ultrasound Med. 22, 207 (2003).

${ }^{7}$ T. Kikuchi, S. Sato, and M. Yoshioka, IEEE International Ultrasonics Ferroelectrics Frequency Control Joint 50th Anniversary Conference (2004), pp. 2197-2200.

${ }^{8}$ K. M. Swamy and F. J. Kail, Ultrason. Sonochem. 9, 305 (2002).

${ }^{9}$ V. N. Bindal and A. Kumar, Acustica 46, 223 (1980).

${ }^{10}$ V. N. Bindal, A. Kumar, and, R. C. Chivers, Acustica 53, 219 (1983).

${ }^{11}$ K. Beissner, Metrologia 36, 313 (1999).

${ }^{12}$ IEC Standard 1157 (61157), International Electrotechnical Commission (1992).

${ }^{13}$ IEC Standard 1689 (61689), International Electrotechnical Commission (1996).

${ }^{14}$ IEC Standard 1161 (61161), International Electrotechnical Commission (1998).

${ }^{15}$ K. Beissner, J. Acoust. Soc. Am. 76, 1505 (1984).

${ }^{16}$ S. E. Fick and F. R. Breckenridge, J. Res. Natl. Inst. Stand. Technol. 101,
659 (1996).

${ }^{17}$ B. Zeqiri, in Output Measurements for Medical Ultrasound, edited by R. C. Preston (Springer-Verlag, London, 1991) Chap. 3, pp. 41-44.

${ }^{18}$ V. A. Del Grosso and C. A. Mader, J. Acoust. Soc. Am. 52, 1442 (1972).

${ }^{19}$ National Physical Laboratory, Kaye and Laby Tables of Physical and Chemical Constants (2005).

${ }^{20}$ S. Rajagopalan, S. J. Sharma, and P. K. Dubey, Rev. Sci. Instrum. 78, 085104 (2007)

${ }^{21}$ Sartorius: MC21S datasheet.

${ }^{22}$ IEC Standard 1088, International Electro-technical Commission (1991).

${ }^{23}$ American Accurate Components, Inc.: High frequency thermal voltage converters specifications.

${ }^{24}$ See: www.ballantinelabs.com for Ballantine Laboratories Inc., 1395B Thermal voltage converter specifications.

${ }^{25}$ K. Takahshi, H. Sasaki, B. D. Inglis, and M. Klonz, IEEE Trans. Instrum. Meas. 48, 391 (1999).

${ }^{26}$ Yu. F. Pavlenko, V. I. Ogar, and V. S. Klimashevskii, Meas. Tech. 50, 676 (2007).

${ }^{27}$ I. Budovsky, IEEE Trans. Instrum. Meas. 58, 844 (2009).

${ }^{28} \mathrm{~W}$. Guilherme and K. Ihlenfeld, Sens. Actuators, A 115, 1 (2004).

${ }^{29}$ G. Kennedy and B. Davis, Electronic Communication Systems, 4th ed. (McGraw-Hill, New York, 1999), pp. 185-218.

${ }^{30}$ IC LM35, Temperature sensor datasheet.

${ }^{31}$ G. Madelin, B. Hosten, C. Biateau, C. Mougenot, J.-M. Franconi, and E. Thiaudiere, Ultrasonics 43, 769 (2005).

${ }^{32}$ Operational Amplifier: IC OP07 datasheet.

${ }^{33}$ See www.datasheetcatalog.com for diode $1 \mathrm{~N} 4148$ datasheet.

${ }^{34}$ See www.fluke.com for Fluke 8808A multimeter manual.

${ }^{35}$ See www.omega.com for high accuracy PCI data acquisition card (OMBDAQTEMP14A). 